\section{ORIGINAL RESEARCH}

\author{
E. Widjaja \\ S. Geibprasert
}

S. Zarei Mahmoodabadi

N.E. Brown

P. Shannon

\title{
Corroboration of Normal and Abnormal Fetal Cerebral Lamination on Postmortem MR Imaging with Postmortem Examination
}

\begin{abstract}
BACKGROUND AND PURPOSE: The presence of normal fetal cerebral lamination of the germinal matrix, intermediate zone, subplate layer, and cortex can be used as a marker of normal fetal cerebral development. Our aim was to compare postmortem MR imaging assessment of normal and abnormal fetal cerebral lamination on T1- and T2-weighted images with histopathology.
\end{abstract}

MATERIALS AND METHODS: Fifty-five formalin-fixed brains from postmortem fetuses, ranging from 16 to 30 weeks' gestational age, mean of 23 weeks, underwent T1- and T2- weighted MR imaging and subsequent sectioning and histologic examination. The cerebral lamination was graded as normal or abnormal on T1- and T2-weighted imaging and compared with postmortem findings. The sensitivity, specificity, and positive and negative predictive values of $\mathrm{T} 1$ and $\mathrm{T} 2$ assessment of cerebral lamination were calculated.

RESULTS: Twenty-six fetuses had abnormal and 29 had normal cerebral lamination on histology. On $\mathrm{T} 1$, the overall sensitivity, specificity, and positive and negative predictive values of evaluating cerebral lamination were $96.15 \%(\mathrm{Cl}, 78.42 \%-99.80 \%)$, 89.66\% (Cl, 71.50\%-97.29\%), 89.29\% (Cl, 70.63\%$97.19 \%)$, and $96.29 \%(\mathrm{Cl}, 79.11 \%-99.80 \%)$, respectively. On T2, the overall sensitivity, specificity, and positive and negative predictive values of evaluating cerebral lamination were $73.08 \%(\mathrm{Cl}$, $51.95 \%-87.65 \%), 96.55 \%(\mathrm{Cl}, 80.37 \%-99.82 \%), 95.00 \%(\mathrm{Cl}, 73.06 \%-99.74 \%)$, and $80.00 \%(\mathrm{Cl}$, $62.54 \%-90.94 \%)$, respectively.

CoNCLusıons: Postmortem MR imaging has high sensitivity, specificity, and positive and negative predictive values in assessing fetal cerebral lamination compared with histology. T1-weighted imaging has a higher sensitivity and negative predictive value, while T2-weighted imaging has a higher specificity and positive predictive value.

ABBREVIATIONS: $\mathrm{C}=$ cortex; $\mathrm{Cl}=$ confidence interval; $\mathrm{G}=$ germinal matrix; I = intermediate zone; $M R I=M R$ imaging; NPV = negative predictive value; PPV = positive predictive value; $S=$ subplate layer

$\mathbf{P}$ ostmortem MR imaging has been used to evaluate the fetal central nervous system and to compare it with postmortem findings. Griffiths et $\mathrm{al}^{1}$ assessed the gross structural morphology of unfixed brain of 40 fetuses from 14 to 42 weeks' gestation by using T2-weighted MR imaging and found that the sensitivity was $100 \%$; specificity, $92 \%$; positive predictive value, 95\%; and negative predictive value, 100\%. Woodward et $\mathrm{al}^{2}$ used 3D T2-weighted imaging to assess unfixed postmortem MR imaging of 26 fetuses and found that the detection rate of major central nervous system and other organ malformations ranged from $79 \%$ to $91 \%$. In both of these studies, the investigators used gross morphology to identify structural abnormalities of the brain on postmortem MR imaging. Although derangement of gross morphology as visualized on MR imaging can be used as a marker of abnormal brain development, it may not be adequate to evaluate the presence

Received March 18, 2010; accepted after revision April 30.

From the Department of Diagnostic Imaging (E.W., S.G., S.Z.M., N.E.B.), Hospital for Sick Children, Toronto, Ontario, Canada; and Department of Pathology (P.S.), Mount Sinai Hospital, Toronto, Ontario, Canada

This work was supported by the Ontario Federation for Cerebral Palsy and the Department of Medical Imaging, University of Toronto, Seed Grant.

Please address correspondence to Elysa Widjaja, MD, Department of Diagnostic Imaging, Hospital for Sick Children, 555 University Ave, Toronto, Ontario M5G 1X8, Canada; e-mail: Elysa.Widjaja@sickkids.ca

DOI 10.3174/ajnr.A2193 of aberration of normal brain development due to immaturity of the brain. Additional markers are needed to assist in the evaluation of the fetal brain on imaging.

Transient fetal cerebral lamination is seen during normal development and could be used as a marker of the presence of normal cerebral development. Laminar organization of the normal fetal brain has been described on T1-weighted imaging on postmortem MR imaging, which correlated with histologic findings. ${ }^{3-5}$ Abnormal laminar organization of the fetal cerebrum has previously been reported on postmortem T1- and T2-weighted MR imaging in a 28-week fetus with cobblestone complex. ${ }^{6}$ The findings on postmortem MR imaging corresponded with histology at postmortem examination. The case highlights the potential of using laminar organization to identify the presence of neuronal migration disorders in the fetus. However, the sensitivity and specificity of MR imaging assessment of normal and abnormal laminar organization of the fetal brain have not been reported.

Our primary hypothesis was that postmortem MR imaging has a high sensitivity and specificity in the assessment of normal and abnormal fetal cerebral lamination and, therefore, can be used as an additional marker of the presence of normal or disrupted development of the cerebral hemispheres. Our secondary hypotheses were as follows: first, postmortem MR imaging has high sensitivity and specificity in the evaluation of fetal cerebral sulcation and gyration; second, abnormal fetal 

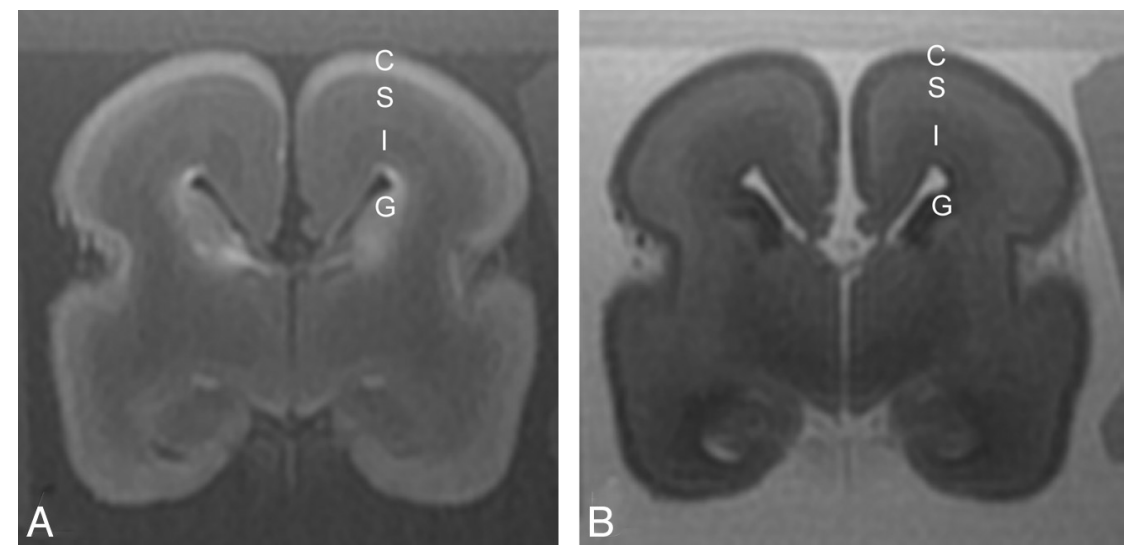

Fig 1. A 22-week fetus with normal cerebral lamination. $A$, On a T1-weighted image, the germinal matrix is of high T1 signal intensity, the intermediate zone is of slightly lower T1 signal intensity relative to the subplate layer, and the cortex is of high T1 signal intensity. B, On a T2-weighted image, the germinal matrix is of low T2 signal intensity, the intermediate zone is of lower T2 signal intensity relative to the subplate layer, and the cortex is of lower T2 signal intensity compared with subplate layer.

cerebral lamination was associated with abnormal sulcation and gyration on postmortem examination. Our primary aim was to compare the postmortem MR imaging assessment of fetal cerebral lamination on T1- and T2-weighted images with histopathology. The secondary aims were to compare postmortem MR imaging assessment of the sulcation and gyration with postmortem examination and also to determine if there was an association of fetal cerebral lamination with sulcation and gyration on postmortem examination.

\section{Materials and Methods}

This prospective study was approved by the institutional research ethics board, and written informed consent was obtained from parents. The inclusion criteria for the postmortem study were fetuses between 16 and 30 gestational weeks undergoing postmortem examination. The exclusion criterion was significant tissue autolysis. The gestational age was estimated on the basis of maternal last menstrual period and sonographic assessment at approximately 12 weeks gestation. Fifty-five fetuses from 16 to 30 weeks gestational age, mean of 23 weeks, were included in the study.

\section{Postmortem MR Imaging}

Postmortem MR imaging was performed by using a 1.5T CV/I magnet (GE Healthcare, Milwaukee, Wisconsin) maximum gradient amplitude and slew rates of $40 \mathrm{mT} / \mathrm{m}$ and $150 \mathrm{~T} / \mathrm{m} / \mathrm{s}$, respectively. Postmortem MR imaging was performed with an 8-channel knee coil following dissection and tissue fixation. Axial, sagittal, and coronal spin-echo T1-weighted $(\mathrm{TR}=500 \mathrm{~ms}, \mathrm{TE}=10 \mathrm{~ms}, \mathrm{FOV}=16 \mathrm{~cm}$, section thickness $=2 \mathrm{~mm}$, matrix $=320 \times 256$; scanning time $=21$ minutes 36 seconds), T2-weighted $(\mathrm{TR}=4115 \mathrm{~ms}, \mathrm{TE}=115 \mathrm{~ms}$, $\mathrm{FOV}=16 \mathrm{~cm}$, section thickness $=2 \mathrm{~mm}$, matrix $=320 \times 256$; scanning time $=5$ minutes $)$, and axial 3D T1-weighted $(\mathrm{TR}=23 \mathrm{~ms}$, $\mathrm{TE}=8 \mathrm{~ms}, \mathrm{FOV}=18 \mathrm{~cm}$, section thickness $=1.0 \mathrm{~mm}$, matrix $=$ $256 \times 256$; scanning time $=18$ minutes 54 seconds) images were obtained in all cases.

The germinal matrix, intermediate zone, subplate layer, and cortex of the cerebral hemispheres were graded as normal (Fig 1) or abnormal by 2 neuroradiologists blinded to the postmortem findings by consensus (E.W. and S.G.) on both spin-echo T1- and T2weighted imaging. The germinal matrix, intermediate zone, subplate layer, and cortex of the cerebral hemispheres were graded as abnormal if the signal intensity or thickness was abnormal or a distinct layer could not be identified. If $\geq 1$ of the cerebral laminations was considered to be abnormal, the overall $\mathrm{T} 1$ and $\mathrm{T} 2$ cerebral lamination was considered to be abnormal. The sulcation and gyration pattern was defined as normal or abnormal compared with the atlas by FeessHiggins and Larroche. ${ }^{7}$

\section{Gross Pathology Section and Histology}

The fetal brains were removed at postmortem examination and immersion-fixed for 14 days in formalin $/ 5 \%$ acetic acid. Sectioning of the fixed brain was performed after MR imaging examination. Histology was performed by using standard paraffin embedding and sectioning at $6 \mu \mathrm{m}$ in the coronal plane to match the coronal postmortem MR imaging. Routine sections in all cases included the frontal and occipital poles, the frontal and parietal centrum semiovale with adjacent cortical structures, the basal ganglia and temporal lobes, and the third ventricle with the thalamus and basal ganglia, as well as any abnormal telencephalic structures. Whole mount was performed by using agar stabilization. ${ }^{8}$ Staining was done with hematoxylin-eosin or cresyl violet. Histology sections were reviewed by a neuropathologist (P.S.), and the germinal matrix, intermediate zone, subplate layer, and cortex were graded as normal or abnormal. The sulcation and gyration patterns were also defined as normal or abnormal compared with the atlas by Feess-Higgins and Larroche. ${ }^{7}$

\section{Statistical Analysis}

MR imaging assessment of the germinal matrix, intermediate zone, subplate layer, cortex, and overall fetal cerebral lamination on both spin-echo T1- and T2-weighted imaging was compared with histopathologic evaluation. MR imaging assessment of sulcation and gyration patterns was also compared with postmortem evaluation. The sensitivity, specificity, and positive and negative predictive values and their confidence intervals were calculated. Spin-echo T1 assessment of cerebral lamination was compared with axial 3D T1 assessment of cerebral lamination by using the McNemar test. $\chi^{2}$ analysis was performed between overall fetal cerebral lamination and sulcation and gyration as evaluated at postmortem examination. $P$ value $<$ .05 was considered statistically significant.

\section{Results}

Of the 55 fetuses evaluated, 26 had overall abnormal cerebral lamination and 29 had normal cerebral lamination as defined by histology. The mean age of fetuses with abnormal cerebral 


\begin{tabular}{|c|c|c|c|c|}
\hline & \multicolumn{2}{|c|}{$\begin{array}{l}\text { T1-Weighted } \\
\text { Images }\end{array}$} & \multicolumn{2}{|c|}{$\begin{array}{c}\text { T2-Weighted } \\
\text { Images }\end{array}$} \\
\hline & Abnormal & Normal & Abnormal & Normal \\
\hline rminal matrix & $20 / 25$ & $29 / 30$ & $19 / 25$ & $30 / 30$ \\
\hline Intermediate zone & $23 / 26$ & $26 / 29$ & $18 / 26$ & $28 / 29$ \\
\hline Subplate layer & $22 / 26$ & $27 / 29$ & $18 / 26$ & $28 / 29$ \\
\hline Cortex & $21 / 26$ & $29 / 29$ & $15 / 26$ & $29 / 29$ \\
\hline Overall cerebral lamination & $25 / 26$ & $26 / 29$ & $19 / 26$ & $28 / 29$ \\
\hline
\end{tabular}

lamination was 23.8 weeks (range, 16-30 weeks), and the mean age of fetuses with normal cerebral lamination was 22.4 weeks (range, 17-30 weeks). Of the 26 fetuses that had abnormal overall cerebral lamination on histology, all 26 cases had an abnormal intermediate zone, subplate layer, and cortex and 25 fetuses had an abnormal germinal matrix. Of those with abnormal cerebral lamination, 18 had bilateral abnormal cerebral lamination and 8 had unilateral abnormal cerebral lamination. The underlying diagnoses of those with abnormal cerebral lamination were as follows: Seven had neuronal migration disorders, 6 had marked ventriculomegaly with associated thinning of the cerebral mantle, 5 had thanatophoric dwarfism, 2 had holoprosencephaly, 2 had encephalocele, 2 had neuroaxonal dystrophy, and 2 had hypoxic-ischemic injury.

T1-weighted imaging correctly identified abnormal overall cerebral lamination in 25/26 (96.15\%) cases and normal overall cerebral lamination in 26/29 (89.66\%) cases (Table 1). T2weighted imaging correctly identified abnormal overall cerebral lamination in 19/26 (73.08\%) cases and normal overall cerebral lamination in 28/29 (96.55\%) cases (Table 1). The sensitivity, specificity, and positive and negative predictive values of MR imaging evaluation of the germinal matrix, intermediate zone, subplate layer, and cortex on both T1-and T2-weighted images are shown on Tables 2 and 3, respectively. Overall, T1 had a higher sensitivity and higher negative predictive value compared with T2 in assessing the cerebral lamination, which had a higher specificity and positive predictive value. T1-weighted imaging had high sensitivity and specificity in identifying an abnormal germinal matrix, intermediate zone, subplate layer, and cortex, ranging from $80.00 \%$ to $88.46 \%$ and $89.66 \%$ to $100 \%$, respectively (Figs $2-4$ ). There were 4 cases in which there was disagreement between overall T1-weighted imaging assessment of cerebral lamination and histology. Three of these cases were false-positive in that T1weighted images demonstrated abnormal cerebral lamination and histology showed normal cerebral lamination. One 25week fetus had patchy areas of high T1 signal intensity in the intermediate zone and subplate layer in both cerebral hemispheres, and histology showed karyorrhectic neurons in brain stem nuclei, indicative of hypoxic-ischemic injury in the brain stem but normal cerebral lamination in both cerebral hemispheres (Fig 5). The second case was a 20-week fetus with Chiari II malformation. Postmortem MR imaging demonstrated lower T1 signal intensity in the intermediate zone compared with the subplate layer diffusely; histology showed normal cerebral lamination. The third case was a 23 -week fetus with foci of high T1 signal intensity scattered in the intermediate zone on postmortem MR imaging; histology demonstrated normal cerebral lamination. The fourth was a falsenegative case in a 30-week fetus whose T1-weighted images showed normal cerebral lamination, and histopathology demonstrated the presence of abnormal cerebral lamination secondary to neuronal-migration disorder affecting the frontal, temporal, and perisylvian regions bilaterally. There were no significant differences between spin-echo T1 assessment of the germinal matrix, intermediate zone, subplate layer, cortex, and overall cerebral lamination compared with 3D T1weighted images $(P>.05)$.

T2-weighted imaging had a moderate-to-high sensitivity of identifying abnormal cerebral lamination, ranging from $57.69 \%$ to $76.00 \%$, and a high specificity in identifying abnormal lamination, ranging from $96.55 \%$ to $100 \%$. There were 8 cases in which there was disagreement between T2-weighted imaging assessment of overall cerebral lamination and histopathology. Seven cases were false-negative in that T2-weighted images showed normal cerebral lamination but histopathology demonstrated abnormal cerebral lamination affecting the germinal matrix, intermediate zone, subplate layer, and cortex diffusely in both cerebral hemispheres. The gestational ages of these cases varied from 18 to 30 weeks. The eighth case was a false-positive one in a 25-week fetus whose T2-weighted images showed patchy areas of lower T2 signal intensity in the intermediate zone and subplate layer in both cerebral hemispheres, and histopathology demonstrated karyorrhectic neurons in brain stem nuclei, indicative of hypoxic-ischemic injury but normal cerebral lamination (Fig 5).

Postmortem MR imaging correctly identified abnormal sulcation and gyration in 25/25 (100\%) and normal sulcation and gyration in $29 / 30(96.67 \%)$ cases. The sensitivity, specific-

\begin{tabular}{|c|c|c|c|c|}
\hline & Sensitivity & Specificity & PPV & NPV \\
\hline T1 germinal matrix & $\begin{array}{l}80.00 \% \\
(\mathrm{Cl}, 58.70 \%-92.39 \%)\end{array}$ & $\begin{array}{l}96.67 \% \\
(\mathrm{Cl}, 80.95 \%-99.83 \%)\end{array}$ & $\begin{array}{l}95.24 \% \\
(\mathrm{Cl}, 74.13 \%-99.75 \%)\end{array}$ & $\begin{array}{l}85.29 \% \\
(\mathrm{Cl}, 68.17 \%-94.46 \%)\end{array}$ \\
\hline T1 intermediate zone & $\begin{array}{l}88.46 \% \\
(\mathrm{Cl}, 68.72 \%-96.97 \%)\end{array}$ & $\begin{array}{l}89.66 \% \\
\text { (Cl, } 71.50 \%-97.29 \%)\end{array}$ & $\begin{array}{l}88.00 \% \\
(\mathrm{Cl}, 68.72 \%-96.97 \%)\end{array}$ & $\begin{array}{l}89.66 \% \\
(\mathrm{Cl}, 71.50 \%-97.29 \%)\end{array}$ \\
\hline T1 subplate layer & $\begin{array}{l}84.62 \% \\
(\mathrm{Cl}, 64.27 \%-94.95 \%)\end{array}$ & $\begin{array}{l}93.10 \% \\
(\mathrm{Cl}, 75.79 \%-98.80 \%)\end{array}$ & $\begin{array}{l}91.67 \% \\
(\mathrm{Cl}, 71.53 \%-98.54 \%)\end{array}$ & $\begin{array}{l}87.10 \% \\
(\mathrm{Cl}, 69.24 \%-95.78 \%)\end{array}$ \\
\hline T1 cortex & $\begin{array}{l}80.77 \% \\
(\mathrm{Cl}, 60.02 \%-92.69 \%)\end{array}$ & $\begin{array}{l}100 \% \\
(\mathrm{Cl}, 85.44 \%-100 \%)\end{array}$ & $\begin{array}{l}100 \% \\
(\mathrm{Cl}, 80.76 \%-100 \%)\end{array}$ & $\begin{array}{l}85.29 \% \\
(\mathrm{Cl}, 68.17 \%-94.46 \%)\end{array}$ \\
\hline T1 overall & $\begin{array}{l}96.15 \% \\
(\mathrm{Cl}, 78.42 \%-99.80 \%)\end{array}$ & $\begin{array}{l}89.66 \% \\
(C), 71.50 \%-97.29 \%)\end{array}$ & $\begin{array}{l}89.29 \% \\
(\mathrm{Cl}, 70.63 \%-97.19 \%)\end{array}$ & $\begin{array}{l}96.29 \% \\
(\mathrm{Cl}, 79.11 \%-99.80 \%)\end{array}$ \\
\hline
\end{tabular}




\begin{tabular}{|c|c|c|c|c|}
\hline & Sensitivity & Specificity & PPV & NPV \\
\hline T2 germinal matrix & $\begin{array}{l}76.00 \% \\
(\mathrm{Cl}, 54.48 \%-89.84 \%)\end{array}$ & $\begin{array}{l}100 \% \\
(\mathrm{Cl}, 85.87 \%-100 \%)\end{array}$ & $\begin{array}{l}100 \% \\
(\mathrm{Cl}, 79.08 \%-100 \%)\end{array}$ & $\begin{array}{l}83.33 \% \\
(\mathrm{Cl}, 66.53 \%-93.04 \%)\end{array}$ \\
\hline T2 intermediate zone & $\begin{array}{l}69.23 \% \\
(\mathrm{Cl}, 48.10 \%-4.91 \%)\end{array}$ & $\begin{array}{l}96.55 \% \\
(\mathrm{Cl}, 80.37 \%-99.82 \%)\end{array}$ & $\begin{array}{l}94.74 \% \\
(\mathrm{Cl}, 71.89 \%-99.72 \%)\end{array}$ & $\begin{array}{l}77.78 \% \\
(\mathrm{Cl}, 60.41 \%-89.27 \%)\end{array}$ \\
\hline T2 subplate layer & $\begin{array}{l}69.23 \% \\
(\mathrm{Cl}, 48.10 \%-84.91 \%)\end{array}$ & $\begin{array}{l}96.55 \% \\
(\mathrm{Cl}, 80.37 \%-88.82 \%)\end{array}$ & $\begin{array}{l}94.74 \% \\
(C l, 71.89 \%-99.72 \%)\end{array}$ & $\begin{array}{l}77.78 \% \\
(\mathrm{Cl}, 60.41 \%-89.27 \%)\end{array}$ \\
\hline T2 cortex & $\begin{array}{l}57.69 \% \\
(\mathrm{Cl}, 37.19 \%-76.03 \%)\end{array}$ & $\begin{array}{l}100 \% \\
(\mathrm{Cl}, 85.44 \%-100 \%)\end{array}$ & $\begin{array}{l}100 \% \\
(\mathrm{Cl}, 74.65 \%-100 \%)\end{array}$ & $\begin{array}{l}72.50 \% \\
(\mathrm{Cl}, 55.86 \%-84.86 \%)\end{array}$ \\
\hline T2 overall & $\begin{array}{l}73.08 \% \\
(\mathrm{Cl}, 51.95 \%-87.65 \%)\end{array}$ & $\begin{array}{l}96.55 \% \\
(\mathrm{Cl}, 80.37 \%-99.82 \%)\end{array}$ & $\begin{array}{l}95.00 \% \\
(\mathrm{Cl}, 73.06 \%-99.74 \%)\end{array}$ & $\begin{array}{l}80.00 \% \\
(\mathrm{Cl}, 62.54 \%-90.94 \%)\end{array}$ \\
\hline
\end{tabular}
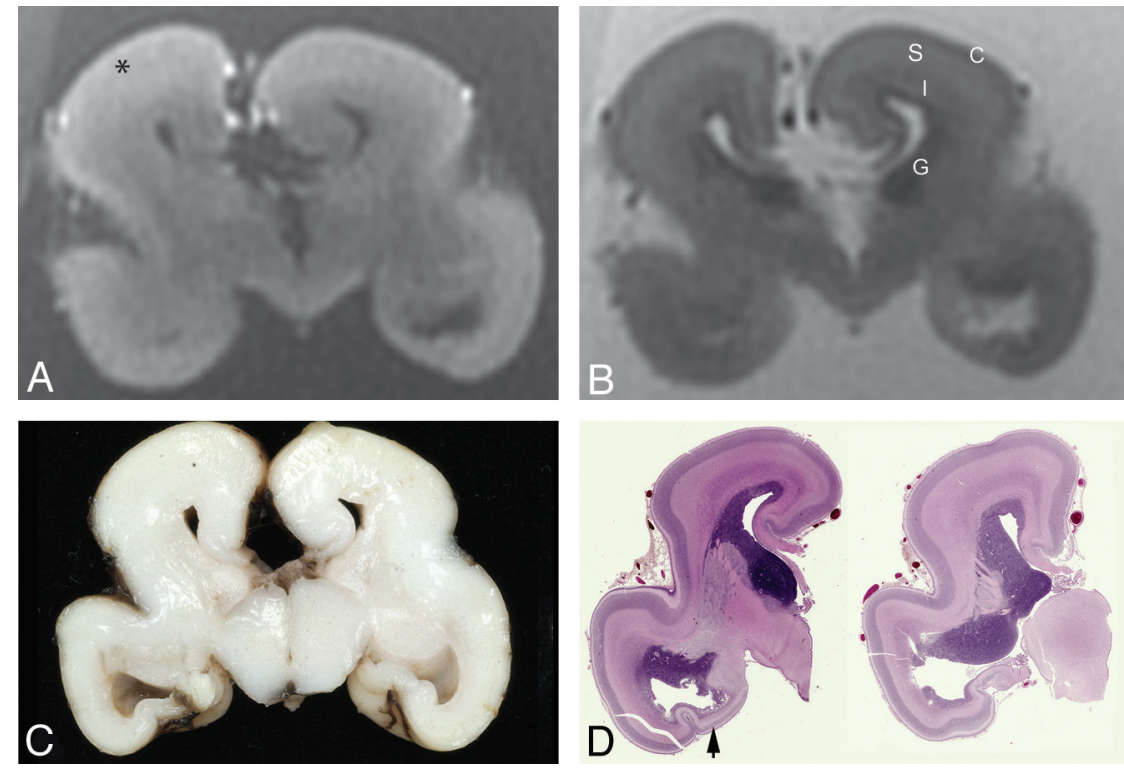

Fig 2. A 22-week fetus with neuroaxonal dystrophy. A, Coronal T1-weighted image shows disruption of normal cerebral lamination, with loss of distinction between the cortex and subplate layer (asterisk), and the high T1 signal intensity of the germinal matrix is not visualized. B, Coronal T2-weighted image shows the presence of normal distinct cerebral lamination. The abnormal cerebral lamination is detected on the T1-weighted image $(A)$ but not on the T2-weighted image $(B)$. The sulcation and gyration of the cerebral hemispheres are abnormal. $C$ and $D$, Gross pathology section of the cerebrum $(C)$ and whole mount histology $(D)$ stain (hematoxylin-eosin, $1 \times$ magnification) show the presence of attenuated lamination in the cerebral mantle. The temporal horns are dilated with a thinned temporal cortex (black arrow).

ity, and positive and negative predictive values of MR imaging evaluation of the sulcation and gyration pattern were $100.00 \%$ (CI, 83.68\%-100.00\%), 96.67\% (CI, 80.95\%$99.83 \%$ ), 96.15\% (CI, 78.42\%-99.80\%), and 100\% (CI, $85.44 \%-100.00 \%)$, respectively. Of the 26 cases with abnormal cerebral lamination, 25 had abnormal sulcation and gyration. Of the 29 cases with normal cerebral lamination, 28 had normal sulcation and gyration. There was significant association between fetal cerebral lamination and sulcation and gyration $\left(\chi^{2}=0.891, P<.0001\right)$. One 27 -week fetus had normal sulcation and gyration, but the cerebral lamination was abnormal on both MR imaging and postmortem examination; this fetus had multiple ischemic changes. Another 30-week fetus had a delayed sulcation and gyration pattern but normal cerebral lamination; this fetus also had multiple extracranial anomalies and cerebellar dysplasia.

\section{Discussion}

Normal fetal cerebral lamination has been described on T1weighted imaging from 15 to 26 postovulatory weeks. ${ }^{3-5} \mathrm{Re}-$ cently, we assessed the alteration in the signal intensity of the subplate layer and intermediate zone in fetuses from 18 to 25 weeks and found decreasing T1 signal intensity and increasing T2 signal intensity of the intermediate zone from approximately 22 weeks, which correlated with reduced cellularity, an increase in the thickness of deep projecting fibers, and an increase in acid mucopolysaccharide on histology. ${ }^{9}$ In the subplate layer, there was increasing T1 signal intensity from 20 to 23 weeks and no alteration in the T2 signal intensity, which remained persistently high from 18 to 25 weeks. The increase in $\mathrm{T} 1$ signal intensity of the subplate layer correlated with a reduction in the extracellular matrix, an increase in cellularity, and an increase in stainable cell processes. ${ }^{9}$ The distinct lamination pattern in the cerebral wall gradually disappears after 26 postovulatory weeks. ${ }^{5}$ Disruption of the expected signal intensity within the cerebral lamination on MR imaging can be used as an indicator of disruption of normal development of the fetal cerebrum because the signal intensity within normal fetal cerebral lamination reflects normal migration and changes in cellularity, development, and organization of the fibers and cell processes. In this study, the 26 fetuses with abnormal cerebral lamination had varying underlying causes for the abnormality, including congenital malformations such as neuronal migration disorders, and acquired disorders, such as 

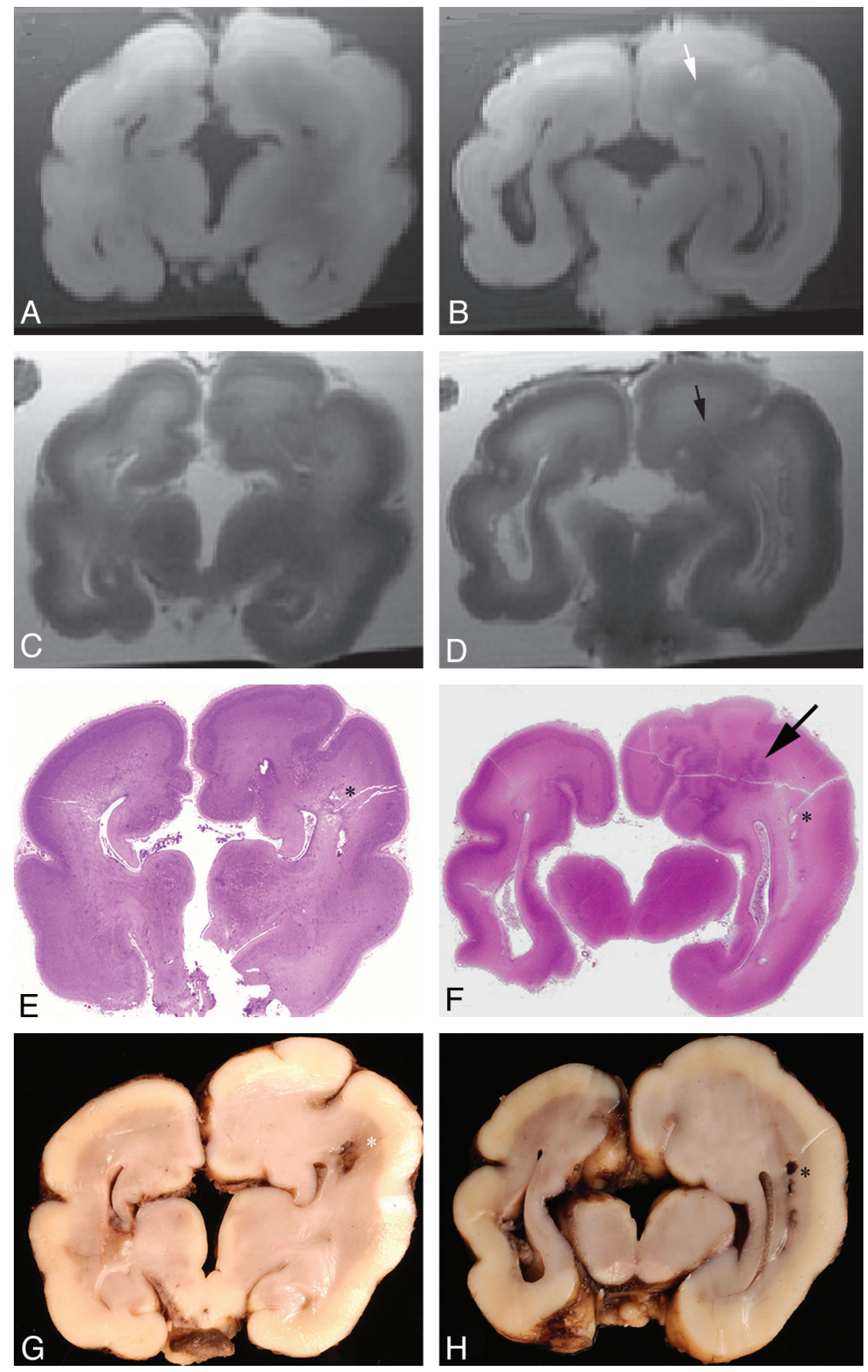

Fig 3. A 29-week fetus with neuronal migration disorder. $A-D$, Coronal T1- $(A$ and $B$ ) and T2-weighted $(C$ and $D)$ images show disruption of the normal cerebral lamination, and the cortical layer appears thickened. There is gray matter heterotopia within the left cerebral mantle (small arrow). The sulcation and gyration are abnormal, and there is also agenesis of the corpus callosum. $E-H$, Whole mount ( $E$ and $A$ stain (hematoxylin-eosin, $1 \times$ magnification) and gross pathology ( $G$ and $H$ ) sections of the brain illustrate agenesis of the corpus callosum and caudate nuclei as well as focal infarcts as indicated by asterisks. There are absence of the normal cerebral lamination and a focal area of deranged cortical architecture within the cerebral mantle (black arrow).

marked ventriculomegaly with thinning of the cerebral mantle. There were 2 fetuses with more severe hypoxic-ischemic changes that had disruption of the cerebral lamination on postmortem MR imaging and histopathology. More severe hypoxic-ischemic injury can lead to disruption of the cerebral lamination, and this could potentially be used as an additional marker of more severe hypoxic-ischemic injury.

We found that T1 assessment of the germinal matrix, intermediate zone, subplate layer, and cortex has higher sensi- tivity and negative predictive value compared with T2. On the other hand, T2 assessment of the cerebral lamination has a higher specificity and positive predictive value. Our findings contrasted with the findings of Woodward et $\mathrm{al}^{2}{ }^{2}$ who found that T1-weighted images showed poor tissue contrast on unfixed postmortem tissues. Current antenatal MR imaging relies heavily on T2-weighted imaging for evaluation of the fetal brain, and T1-weighted imaging is not routinely used due to longer acquisition time, poor signal intensity-to-noise ratio, 

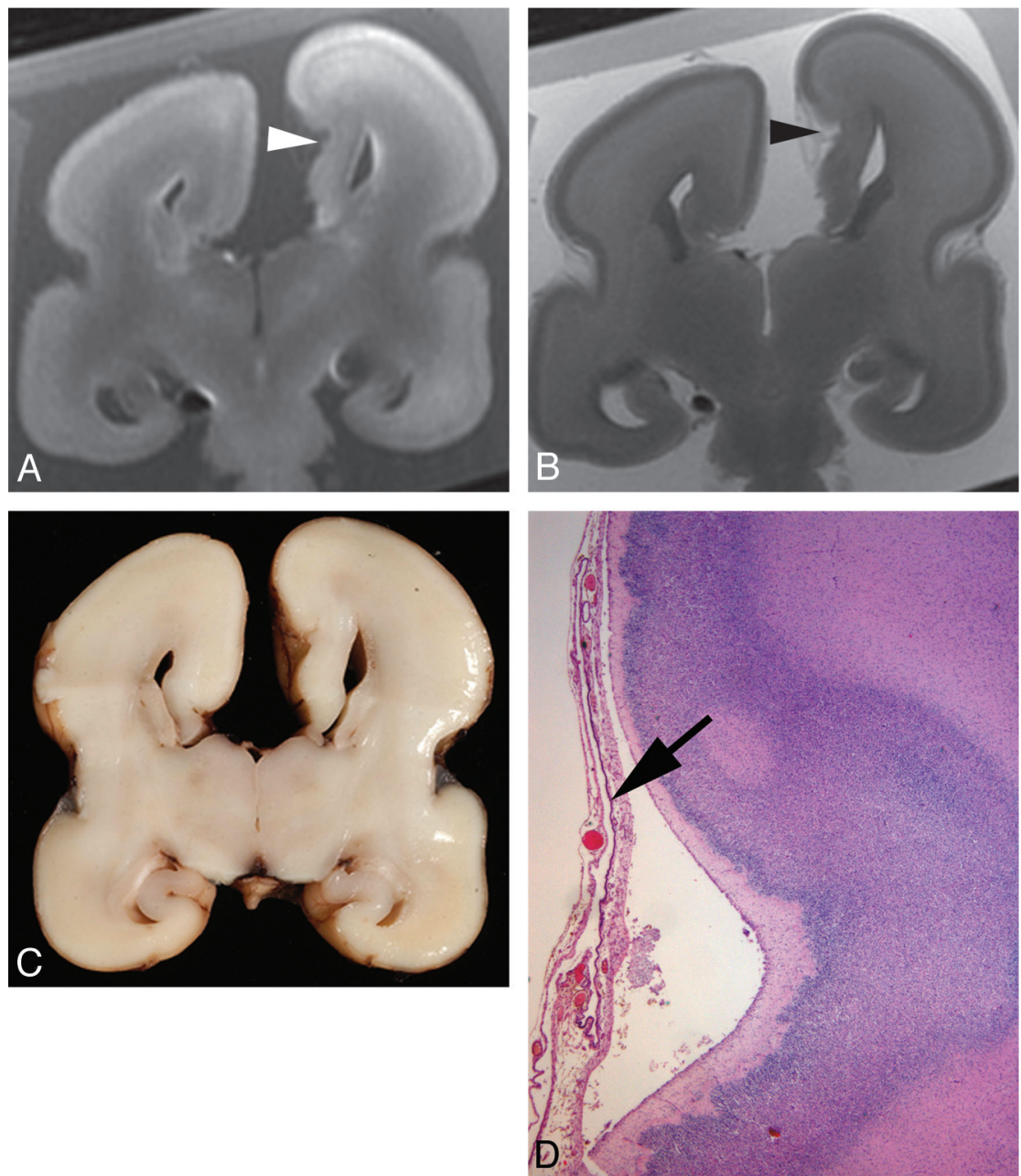

Fig 4. A 22-week fetus with neuronal migration disorder. Coronal T1- $(A)$ and T2-weighted $(B)$ images demonstrate an attenuated cortex, subplate layer, and intermediate zone on the medial aspect of the left frontal lobe, associated with focal deepening of the sulcus at the same site (arrowhead). $C$, Gross pathology section of the cerebrum demonstrates a deep sulcus on the medial aspect of the left frontal lobe. $D$. Histology stain (hematoxylin-eosin, 50× magnification) shows dysplastic hypocellular cortex noted at the site of an abnormally deep sulcus and the overlying arachnoid contains a cyst lined by a single layer of choroid plexus - type epithelium (arrow). There is also agenesis of the corpus callosum.

and greater difficulty in obtaining good-quality imaging. ${ }^{10,11}$ Our findings suggested that T1-weighted imaging contributed to the overall assessment of the fetal cerebral lamination; therefore, T1-weighted imaging should be routinely used to assess the cerebral lamination on antenatal MR imaging. To incorporate ultrafast high-resolution antenatal T1-weighted imaging into routine clinical practice, further research and technical developments on antenatal $\mathrm{T} 1$ sequence are necessary.

There were 3 false-positive cases in which T1-weighted imaging findings were abnormal and findings of histology were normal. One of these false-positive cases was a 23-week fetus with normal cerebral lamination on histology but an abnormal-appearing intermediate zone on postmortem T1weighted MR imaging due to the presence of several foci of higher T1 signal intensity in the intermediate zone. The cause of these high $\mathrm{T} 1$ foci in the intermediate zone was unknown, possibly due to postmortem artifacts leading to false-positive T1-weighted postmortem MR imaging findings. There was 1 false-positive case on both T1- and T2-weighted images. This fetus had mild hypoxic-ischemic changes detectable only in the brain stem and normal cerebral lamination on histology but abnormal cerebral lamination on postmortem MR imag- ing on both T1- and T2-weighted images. The reason for the abnormal cerebral lamination as visualized on postmortem MR imaging was not clear but may possibly be related to subtle changes in tissue water content.

The development of a normal cortical sulcal and gyral pattern is dependent on the successful generation of neuroblasts in the germinal matrix, migration to the cortical surface, and organization within the developing cerebral cortex. ${ }^{12,13} \mathrm{Neu}$ ronal migration affects cortical folding, and the mechanical forces resulting from the cortical folding may also affect the cellular migration during cortical development. ${ }^{14}$ Hence, disruption of cellular migratory processes is associated with abnormal development of sulci and gyri and vice versa. Transient lamination of the fetal cerebrum is related to normal cellular migration. Any congenital or acquired processes that lead to disruption of neuronal migration and normal cerebral lamination can also disrupt normal sulci and gyri development. Therefore, we have found a strong association between fetal cerebral lamination with sulcation and gyration. Most of our cases with abnormal lamination also have abnormal sulcation and gyration. However, normal sulcation and gyration patterns do not necessarily equate to normal cerebral lamination and vice versa. In 1 fetus with a normal sulcation and gyration 

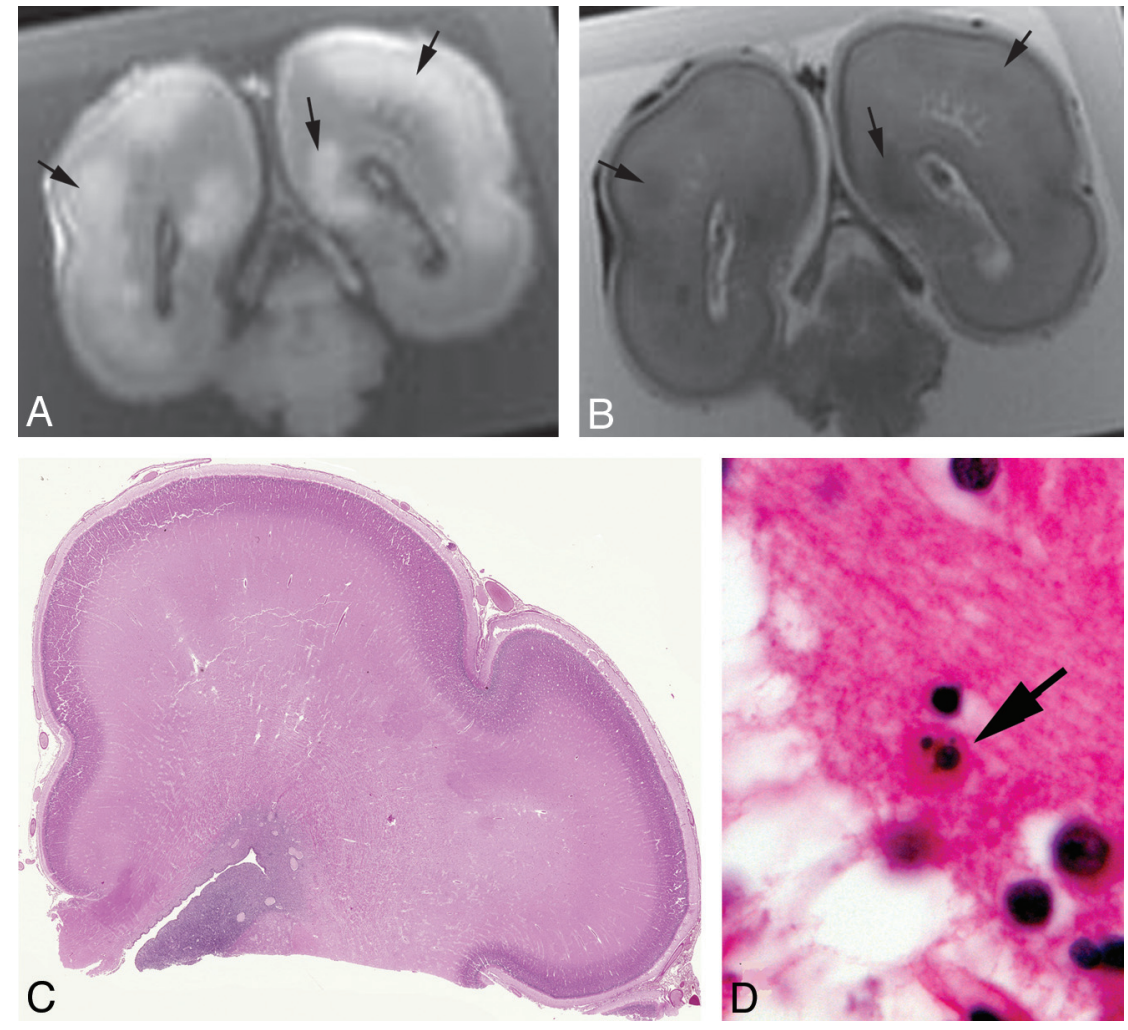

Fig 5. A, 25-week fetus with hypoxic-ischemic injury. Coronal T1- $(A)$ and T2-weighted (B) images demonstrate patchy areas of increased T1 and reduced T2 signal intensity (small arrows) within the subplate layer and intermediate zone. The sulcation and gyration pattern is normal. $C_{r}$ Histology stain (hematoxylin-eosin) demonstrates normal cerebral lamination that is age-appropriate. $D$, Microscopy (630× magnification) demonstrates karyorrhectic neurons (large arrow) in brain stem nuclei, likely due to hypoxic-ischemic injury.

pattern, the cerebral lamination was abnormal on both MR imaging and postmortem examination, and in another fetus with a delayed sulcation and gyration pattern, the cerebral lamination was normal.

\section{Conclusions}

In summary, we found that postmortem MR imaging has high sensitivity, specificity, and positive and negative predictive values in assessing the fetal cerebral lamination compared with histopathology. T1-weighted imaging has higher sensitivity and negative predictive value, while T2-weighted imaging has higher specificity and positive predictive value. Our findings indicate that normal and abnormal fetal cerebral lamination can be assessed with high-resolution MR imaging. This finding is of relevance because fetal cerebral lamination can potentially be a marker of the presence of normal or abnormal cerebral development in fetuses suspected of congenital or acquired disorders. Assessment of fetal cerebral lamination is suboptimal on current antenatal MR imaging, particularly on T1-weighted images, due to poorer resolution and motion degradation of antenatal MR imaging. However, with further technical developments of antenatal MR imaging to improve the resolution and speed of imaging, assessment of fetal cerebral lamination can potentially be applied antenatally as an additional neuroimaging marker of normal and abnormal cerebral development.

\section{References}

1. Griffiths PD, Variend D, Evans M, et al. Postmortem MR imaging of the fetal and stillborn central nervous system. AJNR Am J Neuroradiol 2003;24:22-27

2. Woodward PJ, Sohaey R, Harris DP, et al. Postmortem fetal MR imaging: comparison with findings at autopsy. AJR Am J Roentgenol 1997;168:41-46

3. Chong BW, Babcook CJ, Salamat MS, et al. A magnetic resonance template for normal neuronal migration in the fetus. Neurosurgery 1996;39:110-16

4. Kostovic I, Judas M, Rados M, et al. Laminar organization of the human fetal cerebrum revealed by histochemical markers and magnetic resonance imaging. Cereb Cortex 2002;12:536-44

5. Rados M, Judas M, Kostovic I. In vitro MRI of brain development. Eur J Radiol 2006;57:187-98

6. Widjaja E, Geibprasert S, Blaser S, et al. Abnormal fetal cerebral laminar organization in cobblestone complex as seen on post-mortem MRI and DTI. Pediatr Radiol 2009;39:860-64

7. Feess-Higgins A, Larroche JC. Development of the Human Foetal Brain: An Anatomical Atlas. Paris, France: INSERM Masson; 1987

8. Game M. Embedding in Agar/paraffin. In: Masson P, ed. Histological Techniques. Berlin, Germany: Springer-Verlag; 1976:95

9. Widjaja E, Geibprasert S, Mahmoodabadi SZ, et al. Alteration of human fetal subplate layer and intermediate zone during normal development on MR and diffusion tensor imaging. AJNR Am J Neuroradiol 2010;31:1091-99

10. Rutherford MA. Magnetic resonance imaging of the fetal brain. Curr Opin Obstet Gynecol 2009;21:180-86

11. Glenn OA. MR imaging of the fetal brain. Pediatr Radiol 2010;40:68-81. Epub 2009 Nov 24

12. Barkovich AJ, Gressens P, Evrard P. Formation, maturation, and disorders of brain neocortex. AJNR Am J Neuroradiol 1992;13:423-46

13. Rakic $P$. Neuronal migration and contact guidance in the primate telencephalon. Postgrad Med J 1978;1(54 suppl):25-40

14. Hilgetag CC, Barbas H. Developmental mechanics of the primate cerebral cortex. Anat Embryol (Berl) 2005;210:411-17 